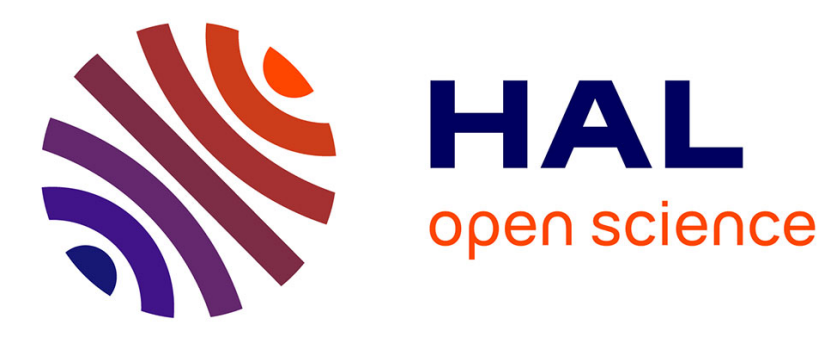

\title{
Soliton complexes in a high power fiber laser
}

\author{
Foued Amrani, Adil Haboucha, Mohamed Salhi, Hervé Leblond, Andrey \\ Komarov, François Sanchez
}

\section{To cite this version:}

Foued Amrani, Adil Haboucha, Mohamed Salhi, Hervé Leblond, Andrey Komarov, et al.. Soliton complexes in a high power fiber laser. Photonics Europe, Solid State Lasers and Amplifiers IV, Apr 2010, Bruxelles, Belgium. pp.77210B-1 - 77210B-8, 10.1117/12.851479 . hal-03192916

\section{HAL Id: hal-03192916 https://univ-angers.hal.science/hal-03192916}

Submitted on 8 Apr 2021

HAL is a multi-disciplinary open access archive for the deposit and dissemination of scientific research documents, whether they are published or not. The documents may come from teaching and research institutions in France or abroad, or from public or private research centers.
L'archive ouverte pluridisciplinaire HAL, est destinée au dépôt et à la diffusion de documents scientifiques de niveau recherche, publiés ou non, émanant des établissements d'enseignement et de recherche français ou étrangers, des laboratoires publics ou privés. 


\title{
Soliton complexes in a high power fiber laser
}

\author{
F. Amrani ${ }^{\text {a }}$, A. Haboucha ${ }^{\text {a }}$, M. Salhi ${ }^{\mathrm{a}}$, H. Leblond ${ }^{\mathrm{a}}$, A. Komarov ${ }^{\mathrm{b}}$, F. Sanchez ${ }^{\text {** }}$ \\ ${ }^{a}$ Laboratoire de Photonique d'Angers EA 4464, Université d'Angers, \\ 2 Bd Lavoisier, 49000 Angers, France \\ ${ }^{\mathrm{b}}$ Institute of Automation and Electrometry, Russian Academy of Sciences, \\ Acad Koptyug Pr. 1, 630090 Novosibirsk, Russia
}

\begin{abstract}
Ordered and disordered pattern formation of solitons is experimentally investigated in the passively mode-locked doubleclad erbium-doped fiber laser. Soliton complexes of about 500 pulses are obtained which organize in different patterns analogous the states of the matter. We have identified a soliton gas, a supersonic soliton gas flow, a soliton liquid, a soliton polycrystal and a crystal of solitons.
\end{abstract}

Keywords: Fiber laser, soliton, short pulses

\section{INTRODUCTION}

Passively mode-locked fiber lasers have demonstrated that they are very good candidates for the investigation of both the generation and the interaction of ultrashort pulses. In particular, double-clad fibers are very useful for high pumping power resulting in short pulse energies [1,2]. The large spectral gain bandwidth favoured short pulse generation while the quantization of the energy in the anomalous dispersion regime leads to the coexistence of many solitons in the cavity, for high enough pumping power. When several solitons are present in the cavity, they interact together, leading to the formation of temporal patterns (or longitudinal spatial patterns if we look at the distribution of the solitons along the cavity). These structures can exhibit some order or can be completely disordered, depending on the nature and on the range of the interaction. Although the existence of forces between solitons was evidenced as early as 1987 [3], it concerned a conservative system. The history of the interaction of dissipative solitons started in 1991 with a paper by Malomed, which predicted the existence of bound solitons [4]. Further works in the nineties mainly concerned theoretical results on bound states of two or a few solitons, using several versions of the complex Ginzburg - Landau equation [5-9]. The first experimental demonstration of a bound state in a passively mode-locked fiber laser was reported in 2001 by Tang et al. [10]. They attributed the formation of such a bound state to a direct interaction between solitons, as suggested by Malomed [2]. In the mean while, other teams reported experimental observation and characterization of bound states in different optical configurations [11-13]. In all cases, theoretical and experimental studies on bound states concerned a few solitons (about 10) [14,15]. The scaling up of the power in fiber lasers has recently permitted the experimental observation of a bound state of hundreds of solitons [16] and, more recently, complex dynamical patterns [17]. Because standard models fail to describe such large number of bounded pulses, alternative models have been recently proposed $[18,19]$. The delay between two neighbouring bound solitons is typically about $10 \mathrm{ps}$ while the bound state remains localized in a part of the cavity even in the case reported in [16], in which the pulse train filled about $10 \%$ of the cavity length. To be complete, it is worth noting that organized structures similar to a soliton crystal have been previously investigated in passive cavities [20,21].

Soliton interaction leads also to the well-known passive harmonic mode-locking, in which the pulses are uniformly distributed along the cavity, resulting in a repetition rate which is multiple of the fundamental cavity frequency (free spectral range) [22]. The interaction of pulses through a cw component has been proposed as responsible for high order harmonic mode-locking [23]. Theoretical confirmation has been done in [24], where it is demonstrated that a small cw component allows to control the attractive or repulsive character and the magnitude of the soliton interaction. Here again,

*francois.sanchez@univ-angers.fr, fax + 33141735216.

Solid State Lasers and Amplifiers IV, and High-Power Lasers, edited by Thomas Graf, Jacob I. Mackenzie,

Helena Jelinková, Gerhard G. Paulus, Vincent Bagnoud, Catherine Le Blanc, Proc. of SPIE Vol. 7721,

77210B · (C) 2010 SPIE · CCC code: 0277-786X/10/\$18 - doi: 10.1117/12.851479

Proc. of SPIE Vol. 7721 77210B-1 
the increase of the power in fiber lasers has allowed the achievement of very high order harmonics $[25,26]$. As in the case considered in the previous paragraph, the harmonic passive mode-locking was an ordered state, but it completely filled the cavity. The distance between the solitons was about a few hundred ps.

States discussed in the previous paragraph are ordered because they repeat indefinitely from one round-trip to the other. States involving many solitons randomly distributed inside the cavity also occur. They have been observed in many cases with various optical configurations and they are often named bunch states. Their characteristic is that each soliton moves relative to the others, and that the soliton set fills the cavity when the pumping power is high enough. This behaviour is of poor practical interest and, as a consequence, has been few studied. However, in the case of a passive cavity, such states have been investigated by analogy with a gas (soliton gas) and even cooling was achieved by spectral filtering $[20,21,27]$. Especially, it has been theoretically shown by Rutz et al. [28] that freezing of the gas and melting of the solid could be realized by means of band pass and band reject filters, respectively. The perpetual movement of solitons was due to the fact that their central frequencies were slightly different, resulting in slightly different group velocities.

The aim of this paper is to present an extensive characterization of the output behaviours observed in passively modelocked double-clad erbium-doped fiber lasers operating in the anomalous dispersion regime and emitting hundreds solitons by cavity round-trip. We have identified 5 different states which will be characterized through temporal evolution, optical spectrum and autocorrelation trace. We point out an analogy with the states of the matter and propose for the first time a classification of dissipative soliton complexes: soliton gas, supersonic soliton gas flow, soliton liquid, soliton polycrystal and soliton crystal. The transition from one state to the other is controlled, for given experimental conditions, by the phase plates. Although part of this terminology has been previously introduced in passive cavities for the description of the transition from a soliton gas to a soliton crystal $[20,21,27,28]$, the large number of available solitons in our cavity allow us to identify new states such as supersonic gas flow, liquid and polycrystal. In the case of disordered states, a statistical approach has been applied in order to extract information about the distribution of the solitons along the cavity.

\section{EXPERIMENTAL RESULTS}

\subsection{Experimental setup}

The experimental setup is shown in Fig.1 [16,18]. Mode locking is achieved through the combined action of nonlinear polarization evolution occurring in the fibers and the discrimination of polarization states by an intracavity polarizer [29]. The laser cavity is a unidirectional ring which includes several fibers. We use a double-clad Er:Yb fiber amplifier manufactured by Keopsys and operating at $1.55 \mu \mathrm{m}$. Two identical laser diodes operating at $980 \mathrm{~nm}$ and emitting about 3 $\mathrm{W}$ each are used in a counter-propagating geometry. The v-groove technique is used to launch intense pump beams into the inner cladding. The $8 \mathrm{~m}$ long double-clad fiber (DCF) has a second order dispersion $\beta_{2}^{\mathrm{DCF}}=-0.015 \mathrm{ps}^{2} / \mathrm{m}$. Pieces of standard fiber (SMF 28) and dispersion-shifted fiber (DSF, $\beta_{2}^{\mathrm{DSF}}=0.14 \mathrm{ps}^{2} / \mathrm{m}$ ) are used to control the total dispersion of the cavity that is $\beta_{2}^{\mathrm{TOT}} \mathrm{L}=-0.04 \mathrm{ps}^{2}$. The total cavity length is about $21 \mathrm{~m}$ leading to a photon round-trip time $\mathrm{T}=$ $105 \mathrm{~ns}$. Nonlinear losses can be varied by a rotation of the intracavity phase plates placed at each side of the intracavity polarization beam splitter. A $10 \%$ output coupler is used to extract the power from the cavity. The output beam is detected with a high-speed photodiode (Newport TIA $120013 \mathrm{GHz}$ ) and analyzed with either a high-speed oscilloscope (Tektronix TDS 6124C $12 \mathrm{GHz}, 40 \mathrm{GS} / \mathrm{s}$ ) or an electronic spectrum analyzer (Rohde \& Schwarz FSP Spectrum Analyzer $9 \mathrm{kHz}-13.6 \mathrm{GHz}$ ). Pulse duration is measured with an optical autocorrelator (Femtochrome FR-103 XL) with a scanning range scalable to about $170 \mathrm{ps,}$, and an optical spectrum analyzer (Anritsu MS 9710C) is also used. In the following, the pumping power is fixed to $3.3 \mathrm{~W}$ (except for the harmonic mode-locking case for which the stability at such high pumping level is poor) and the nonlinear losses are slightly varied by a rotation of the phase plates. Such rotation does not lead to significant variations of the number of solitons, which is in the range $400-500$ pulses, but greatly affects the distribution of the soliton inside the cavity (the laser threshold does not vary significantly). This suggests that a variation of the nonlinear losses directly changes the nature, the amplitude and the range of the interaction between solitons. Indeed, harmonic mode-locking requires repulsive force with long-range interaction while soliton bounding requires attractive force with moderate interaction range and short-range repulsive force. 


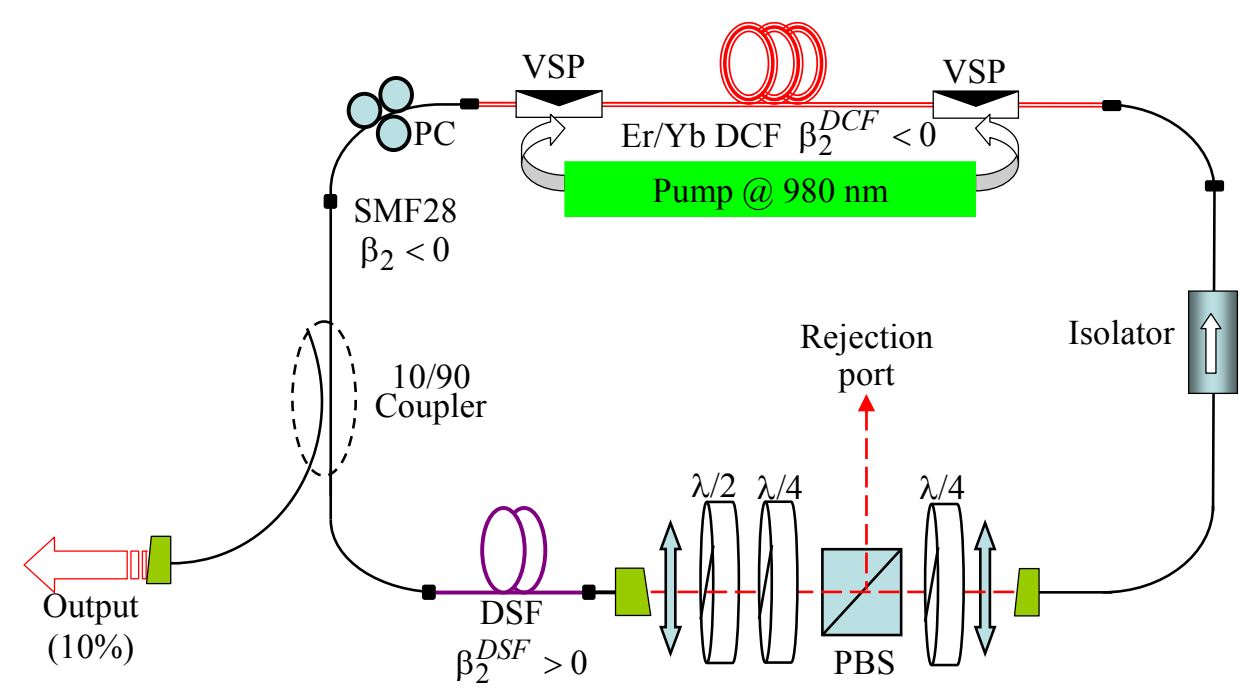

Figure 1. Experimental setup. VSP: v-groove side-pumping. DCF: double-clad fiber. DSF: dispersion shifted fiber. SMF: single-mode fiber. PBS: polarization beam splitter.

\subsection{Gas state}

In our configuration, the most commonly observed state is a distribution in which solitons are disordered along the cavity and in perpetual movement; they occupy all the available space along the cavity. Indeed, in the frame moving at the group velocity, solitons move because of their small differences in group velocity, which may result from different central wavelengths, or eventually from nonlinear or non-conservative effects. This is demonstrated in Fig. 2(a), which gives an example of the time distribution of the solitons (the range is limited to about one round-trip). This state is analogous to a gas and can be seen as a soliton gas. Fig. 2(b) shows the corresponding optical spectrum which, because it is not modulated, proves that there is no constant phase relation between solitons from one round trip to the other (i.e., there is no mutual coherence between pulses). The autocorrelation trace given in Fig. 2(c) exhibits a large pedestal demonstrating the movement of pulses during acquisition time. In addition, the absence of intercorrelation peaks confirms that there is no coherence between the solitons. Hence the solitons move and collide indefinitely. In order to give additional physical insight to this disordered state, we provide in Fig. 2(d) the histogram of the delays between two consecutive solitons $(\Delta \tau)$. It has been extracted from the temporal trace of Fig. 2(a) but recurs approximately from one round-trip to the other. The total number of solitons is 380 per cavity round-trip (note that it is only a lower limit because experimental detection does not allow to distinguish two solitons closer than about $50 \mathrm{ps}$ ). The delays are distributed in a wide range with a mean value of $236 \mathrm{ps}$, large in comparison with the pulse duration which is about $1 \mathrm{ps}$. This suggests that soliton interaction is weak thus allowing the possibility for the pulses to move away from each other. The variance is also large (207 ps) revealing that the distribution of the pulses along the cavity is far from being homogeneous.

\subsection{Supersonic flow}

In this experiment the pumping power is limited to $2.2 \mathrm{~W}$ in order to obtain a stable signal. With a suitable adjustment of the phase plates, the same laser configuration can generate a state in which solitons completely fill the cavity in a homogeneous distribution thus leading to the harmonic mode-locking [22]. In this case, the solitons are well separated and equidistant along the cavity. An example is shown in Fig. 3(a) which gives the temporal trace corresponding to the $322^{\text {nd }}$ harmonic of the fundamental cavity frequency [26]. Because of the transient regime leading to this state is very long $[25,26]$, we can expect that soliton interaction is very weak and practically vanishes at equilibrium. Because the solitons fill the totality of the available space in the cavity, it is analogous to a gas. On the other hand, the relative velocity of solitons vanishes. This situation is therefore very similar to a supersonic atomic beam in which the dispersion of the velocity distribution around the mean value is very small. We can refer to this state as a supersonic soliton gas flow. The transition from a gas to a supersonic gas flow is obtained through cooling (the translational temperature is very low because of the narrowing of the velocity dispersion). Hence, the transition from the soliton gas to a supersonic soliton gas flow can also be interpreted as a soliton cooling (without state changing) in the sense that there is a very strong reduction of the dispersion of the distribution of group velocities. As in the case of a soliton gas, we provide a 
histogram of the delays between two neighbouring pulses $(\Delta \tau)$. The results are shown in Fig. 3(b) and reveal a sharp peak around a mean value of $312 \mathrm{ps.} \mathrm{The} \mathrm{variance} \mathrm{of} 15$ ps lies below the resolution of both the photodetector and the oscilloscope (25 ps), and hence is of little interest. However, it gives an upper limit for the time jitter.
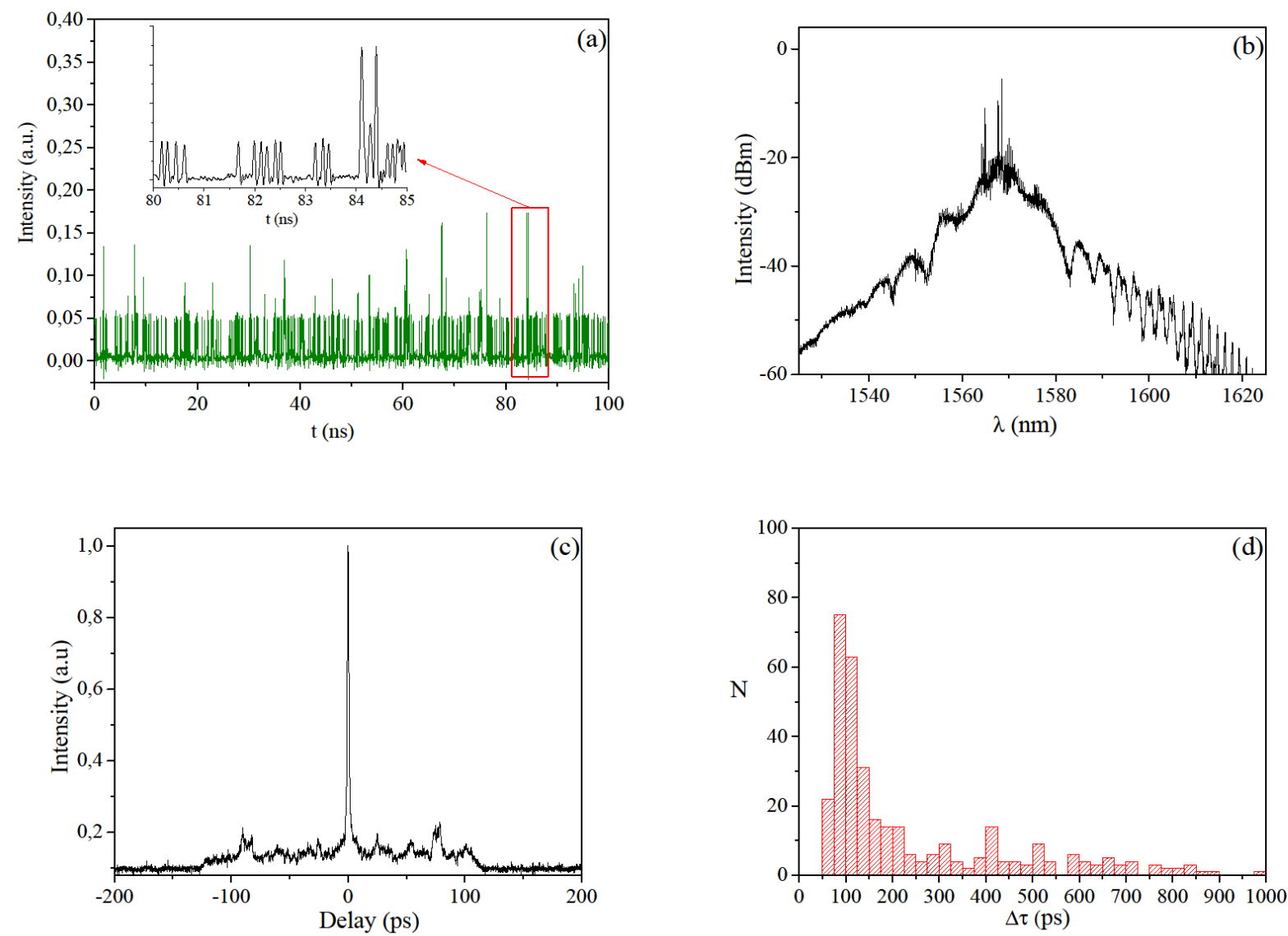

Figure 2. Soliton gas. (a) Time distribution. (b) Optical spectrum. (c) Autocorrelation trace. (d) Histogram of the delays between solitons.
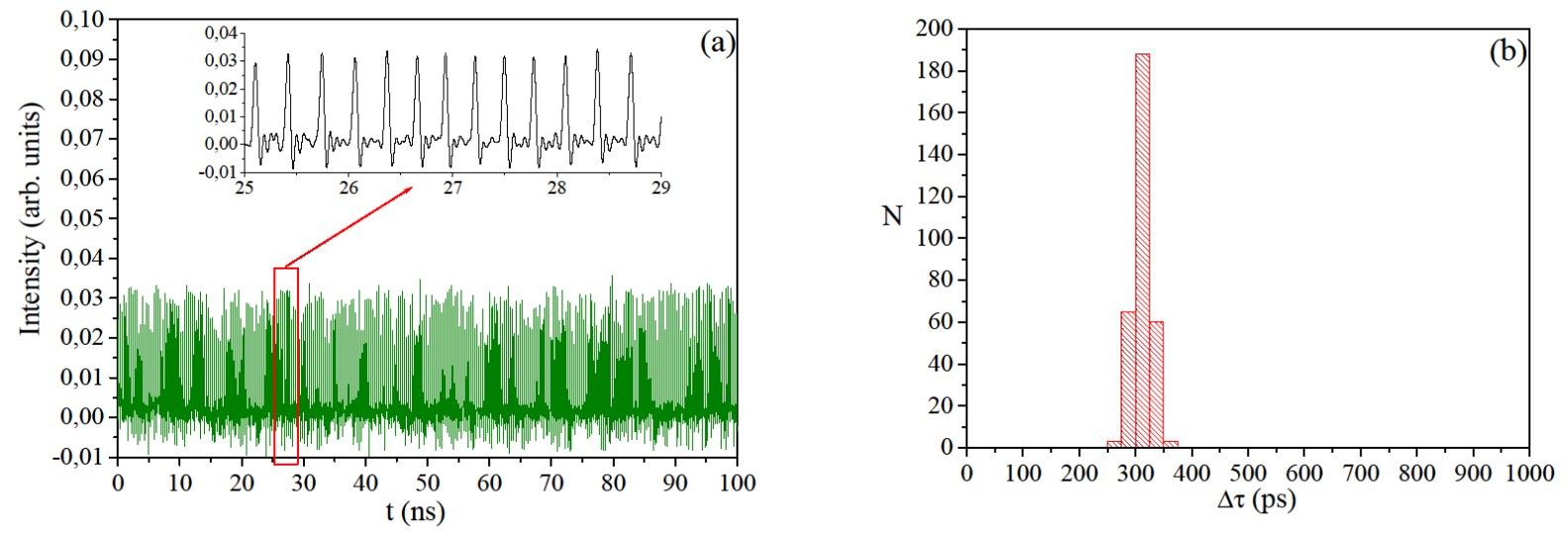

Figure 3. Supersonic soliton gas flow. (a) Temporal trace obtained in the harmonic mode-locked regime. (b) Histogram of the delays between solitons in the case of harmonic mode-locking. 


\subsection{Liquid state}

Starting from a soliton gas, it is possible to obtain some condensate phase shown in Fig. 4(a) by adjusting the phase plates. The temporal trace of Fig. 4(a) reveals that the solitons fill only a small part of the cavity, about $15 \%$. This demonstrates that the system has undergone a phase transition from a gas to a liquid or a solid. Further temporal analysis has shown that solitons or groups of solitons move inside the $15 \mathrm{~ns}$ range. This definitely characterizes the state of the solitons, it corresponds to a liquid state because solitons fill only a part of the available space and are in relative motion inside a definite range. Such state can be conveniently named a soliton liquid. Additional insight about the local structure can be obtained from the optical spectrum and the autocorrelation trace. The optical spectrum (not reported here) points out a small modulation which suggests that a small coherence starts to occur between pulses. This is confirmed by the autocorrelation trace shown in Fig. 4(b) which exhibits some sharp peaks separated by 3 ps revealing that there exist some clusters of solitons, containing typically 5 pulses. The pedestal is characteristic of the fact that solitons and groups of solitons change their location from one round-trip to the other. Hence, the state described here is similar to a liquid of soliton clusters. Because of the perpetual movement of solitons in the $15 \mathrm{~ns}$ range, it is not possible at the present stage of research to extract information concerning the average distance and the ratio of partially coherent solitons. This state is the most difficult to characterize because of the short distance between pulses and their perpetual movement. In addition, bound states of few pulses can be created and destroyed because of the relative motion and soliton interaction.
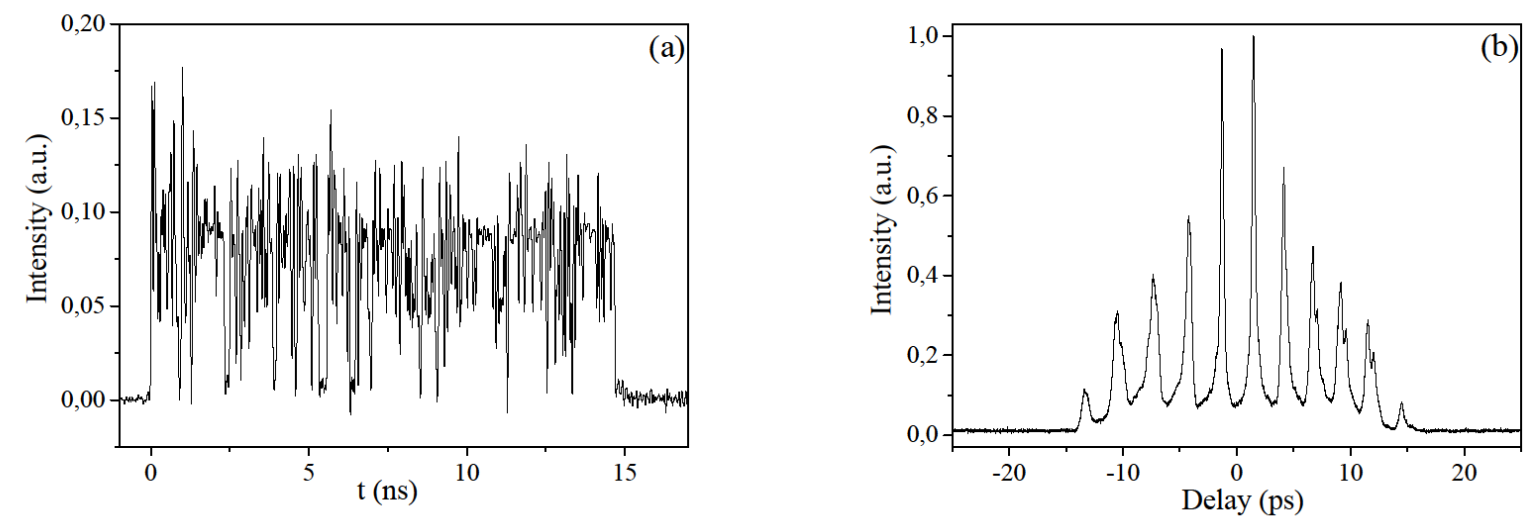

Figure 4. Soliton liquid. (a) Temporal evolution. (b) Autocorrelation trace.

\subsection{Polycrystalline state}

Fig. 5(a) shows another state which can be obtained from the previous one by a slight variation of one halfwave plate. The distribution of pulses occupies a range of about $13 \mathrm{~ns}$ in the cavity. The two insets, which correspond to successive magnifications, show that there is no evidence of a regular structure. In comparison with the liquid state, this state does not change from one round-trip to another. It corresponds to a solid state in which there is no macroscopic periodicity. Therefore we could refer to this state as a soliton glass. Indeed, there is no relative movement of solitons and there is no order at large scale. However, as it will be demonstrated below, there is some local order thus rending the "glass" terminology inappropriate. The latter point can be seen in the optical spectrum shown in Fig. 5(b). The spectrum exhibits a regular modulation revealing some mutual coherence between pulses. From the spectrum period we deduce that the distance between coherent solitons is $23 \mathrm{ps}$. Additional information can be obtained from the autocorrelation trace shown in Fig. 5(c). It exhibits narrow equidistant peaks $(23 \mathrm{ps})$ with an almost triangular envelop which is characteristic of a bound-state $[18,30]$. The range of the autocorrelator does not allow to vizualize all the pulses but a simple extrapolation permits to estimate this number to 8 . In addition, the autocorrelation trace does not point out a pedestal thus confirming that the pulses are not in relative motion. So the soliton glass exhibits an order at the scale of a few pulses but no periodic structure at the scale of the round-trip period. Hence it presents a close analogy to a polycrystalline state, each 8-pulse bound state playing the role of a microcrystal. In this sense, the appellation soliton polycrystal appears to be more relevant.

In addition, some questions remain to be adressed: are there only bound-states of 8 pulses and how many bound-states are inside the global state? It is not easy to answer these questions because of the limitations of our detection apparatus. 
We can however bring a first response if we assume that all possible bound states in the structure involve pulses separated by 23 ps. This is a reasonable assumption because this characteristic time appears as the fundamental one in both the optical spectrum (Fig. 5(b)) and the autocorrelation trace (Fig. 5(c)). On the other hand, from the time series shown in Fig. 5(a) we can identify many packets of solitons. Inside a packet, solitons are close enough to be bounded. So by dividing the length of a given packet by the distance between two bound solitons, say $23 \mathrm{ps,} \mathrm{we} \mathrm{obtain} \mathrm{the} \mathrm{number} \mathrm{of}$ pulses in the bound state. This operation can be repeated for all visible packets inside the 13 ns total range visible in Fig. 5(a). With this procedure we find a total of about 520 pulses in the polycrystalline state. Results are summarized in Fig. 5(d) which gives, through a histogram representation, the number of bound states containing a given number of pulses. For example, we have found 11 bound states containing 5 pulses. The mean value is 8 . Finally, the soliton polycrystal can be viewed as an incoherent superposition of soliton microcrystals containing various numbers of bounded pulses.
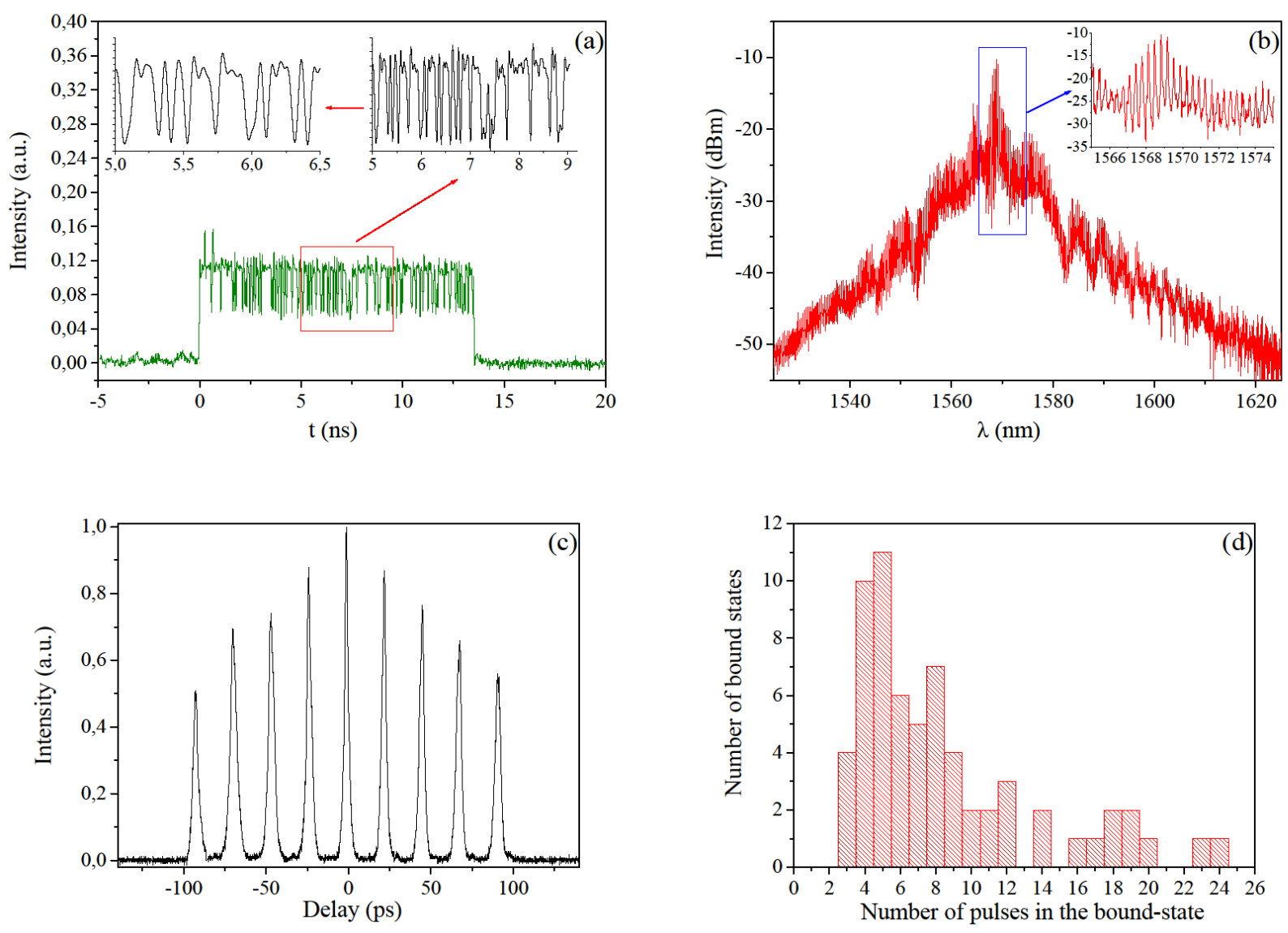

Figure 5. Soliton polycrystal. (a) Temporal evolution. (b) Optical spectrum. (c) Autocorrelation trace. (d) Histogram of the number of pulses in a bound state.

\subsection{Crystal state}

The last state of interest is a unique bound-state with a very large number of pulses analogous to a crystal of finite extent. Such behaviour has been previously reported by our team $[16,18]$ and we will just recall here the main properties for completeness. This fully ordered state was named a soliton crystal by analogy with the results of Mitschke [20,21]. The temporal evolution is represented in Fig. 6(a). The fast oscilloscope gives only the envelope of the signal because pulses cannot be separated. Inspection of the optical spectrum (Fig. 6(b)) and the autocorrelation trace (not reported here) reveals that the signal is composed of hundreds of identical pulses, which are mutually coherent and regularly spaced. This is proved by the strong modulation of the optical spectrum. The autocorrelation trace demonstrates that the pulse separation is 23 ps. The total extent of the soliton crystal is $11 \mathrm{~ns}$, leading to a total number of solitons of about 480 which is actually a record (the number of bound pulses was about 350 in our previous papers $[16,18]$ ). 

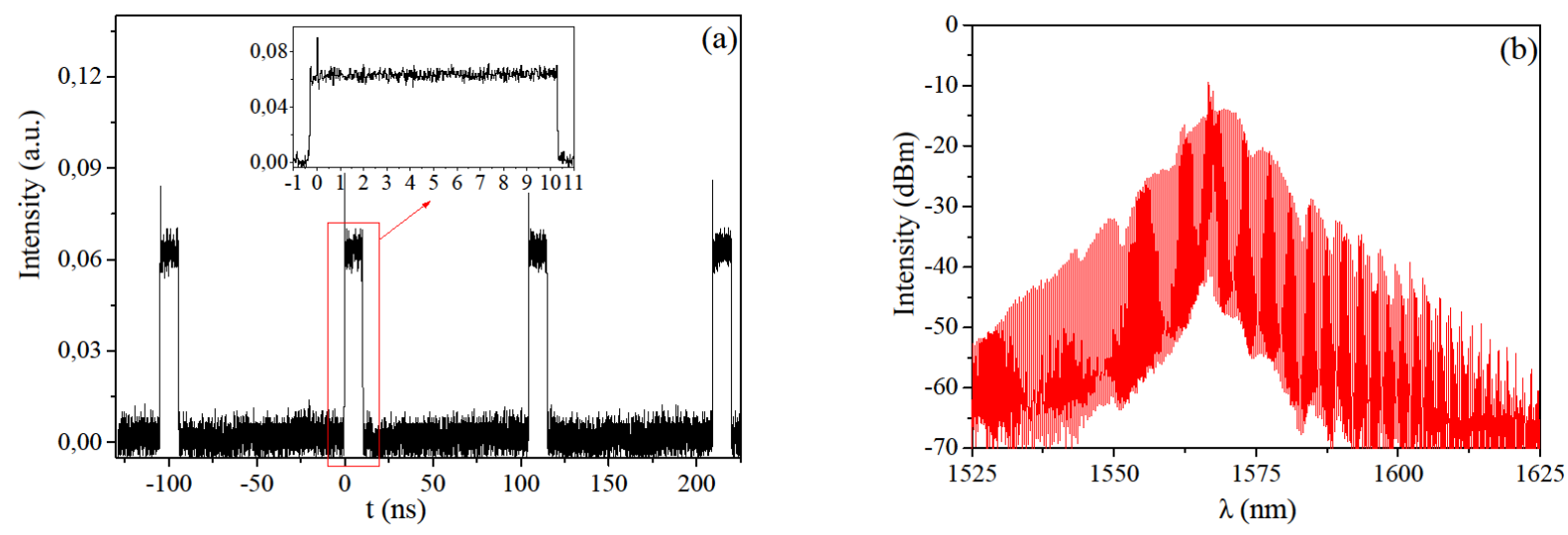

Figure 6. Soliton crystal. (a) Temporal evolution. (b) Optical spectrum.

\section{CONCLUSION}

In this paper, we have investigated the formation of dissipative soliton patterns in a double-clad erbium-doped fiber laser. In particular, we have given a phenomenological classification of the different states. It has appeared that there is a strong analogy between soliton complexes and the states of matter. Indeed, we have pointed out the existence of a soliton gas, a supersonic soliton gas flow, a soliton liquid, a soliton polycrystal and a soliton crystal. Of course, there are also some strong differences, as for example the fact that soliton patterns are one-dimensional, while matter is threedimensional. In spite of that, the results clearly demonstrate that the interactions between a large number of solitons behave in the same way as the interactions between a large number of atoms do, since they lead to very similar patterns formation. Our work is a first important step towards an understanding of the interaction of a large assembly of dissipative solitons. It gives the basis of a physical interpretation and an approach to this very difficult task. Our results open wide perspectives, not only experimentally where new methods have to be invented for the characterization of soliton complexes, but also theoretically where historical models generally fail for the description of hundreds of solitons. A good starting point will be the model proposed in [31] which has demonstrated its ability to generate thousands of pulses in the framework of a bound-soliton harmonic passive mode-locked fiber laser [19].

\section{REFERENCES}

[1] Hideur, A., Chartier, T., Brunel, M., Louis, S., Özkul, C. and Sanchez, F., "Generation of high energy femtosecond pulses from a side-pumped Yb-doped double-clad fiber laser", Appl. Phys. Lett. 79, 3389-3391 (2001).

[2] Ortaç, B., Hideur, A., Chartier, T., Brunel, Özkul, C. and Sanchez, F., "90 fs stretched-pulse ytterbium-doped double-clad fiber laser", Opt. Lett. 28, 1305-1307 (2003).

[3] Mitschke, F. M., and Mollenauer, L. F., "Experimental observation of forces between solitons in optical fibers", Opt. Lett., 12, 355-357 (1987).

[4] Malomed, B. A., "Bound solitons in the nonlinear Schrôdinger-Ginzburg-Landau equation", Phys. Rev. A 44, 6954-6957 (1991).

[5] Malomed, B. A., "Bound states of envelope solitons", Phys. Rev. E 47, 2874-2879 (1993).

[6] Afanasjev, V. V., Malomed, B. A., and Chu, P., "Stability of bound states of pulses in the Ginzburg-Landau equation", Phys. Rev E 56, 6020-6025 (1997).

[7] Afanasjev, V. V., and Akhmediev, N. N., "Soliton interaction and bound states in amplified-damped fiber systems", Opt. Lett. 20, 1970-1972 (1995).

[8] Akhmediev, N. N., Ankiewicz, A. and Soto-Crespo, J. M., "Multisoliton solutions of the complex GinzburgLandau équation", Phys. Rev. Lett. 79, 4047-4051 (1997).

[9] Akhmediev, N. N., Ankiewicz, A. and Soto-Crespo, J. M., "Stable soliton pairs in optical transmission lines and fiber lasers", J. Opt. Soc. Am. B 15, 515-523 (1998). 
[10] Tang, D. Y., Man, W. S., Tam, H. Y. and Drummond, P. D., "Observation of bound states of solitons in a passively mode-locked fiber laser”, Phys. Rev. A 64, 033814 (2001).

[11] Ortaç, B., Hideur, A., Chartier, T., Brunel, Leblond, H. and Sanchez, F., "Ultra-short bound states generation with a passively mode-locked Yb-doped double-clad fiber laser", Opt. Com. 225, 71-78 (2003).

[12] Grelu, Ph., Belhache, F., Gutty, F. and Soto-Crespo, J. M., "Phase-locked soliton pairs in a stretched-pulsed fiber laser", Opt. Lett. 27, 966-968 (2002).

[13] Grelu, Ph., Belhache, F., Gutty, F. and Soto-Crespo, J. M., "Relative phase locking of pulses in a passively modelocked fiber laser", J. Opt. Soc. Am. B 20, 863-870 (2003).

[14] Soto-Crespo, J. M., Akhmediev, N. N., Grelu, Ph., and Belhache, F., "Quantized separations of phase-locked soliton pairs in fiber lasers", Opt. Lett. 28, 1757-1759 (2003).

[15] Akhmediev, N. N., Soto-Crespo, J. M., Grapinet, M. and Grelu, Ph., "Dissipative soliton interactions inside a fiber laser cavity", Opt. Fib. Tech. 11, 209-228 (2005).

[16] Haboucha, A., Leblond, H., Salhi, M., Komarov, A. and Sanchez, F., "Coherent pattern soliton formation in a fiber laser.”, Opt. Lett._33, 524-526 (2008).

[17] Chouli, S. and Grelu, Ph., "Rains of solitons in a fiber laser", Opt. Express 17, 11776 (2009).

[18] Haboucha, A., Leblond, H., Salhi, M., Komarov, A. and Sanchez, F., "Analysis of soliton pattern formation in passively mode-locked fiber laser”, Phys. Rev. A 78, 043806 (2008).

[19] Komarov, A., Haboucha, A. and Sanchez, F., "Ultrahigh repetition rate bound-soliton harmonic passive modelocked laser.", Opt. Lett. 33, 2254-2256 (2008).

[20] Malomed, B. A., Schwache, A. and Mitschke, F., "Soliton lattice and gas in passive fiber-ring resonators", Fib. Int. Opt. 17, 267-277 (1997).

[21] Rutz, S. and Mitschke, F., "Towards thermodynamics of solitons: cooling”, J. Opt. B: Quant. Semiclass. Opt. 2, 364-366 (2000).

[22] Grudinin, A. B. and Gray, S., "Passive harmonic mode locking in soliton fiber lasers", J. Opt. Soc. Am. B. 14, 144-154 (1997).

[23] Zhang, Z. X., Zhan, L., Yang, X. X., Luo, S. Y. and Xia, Y. X., "Passive harmonically mode-locked erbiumdoped fiber laser with scalable repetition rate up to $1.2 \mathrm{GHz}$ ", Laser Phys. Lett. 4, 592-596 (2007).

[24] Komarov, A., Komarov, K., Leblond, H. and Sanchez, F., "Spectral-selective management of dissipative solitons in passive mode-locked fibre lasers", J. Opt. A: Pure Appl. Opt. 9, 1149-1156 (2007).

[25] Ortaç, B., Hideur, A., Martel, G. and Brunel, "2-GHz passive harmonically mode-locked Yb-doped double-clad fiber laser", Appl. Phys. B 81, 507-509 (2005).

[26] Amrani, F., Haboucha, A., Salhi, M., Leblond, H., Komarov, A., Grelu, Ph. and Sanchez, F., "Passively modelocked erbium-doped double-clad fiber laser operating in the 322th harmonic", Opt. Lett. 34, 2120-2122 (2009).

[27] Schwache, A. and Mitschke, F., "Properties of an optical soliton gas", Phys. Rev. A 55, 7720-7725 (1997).

[28] Rutz, S., Körösi, T. and Mitschke, F., "Manipulation of soliton ensembles by spectral filters", Appl. Phys. B 72, 101-104 (2001).

[29] Salhi, M., Leblond, H. and Sanchez, F., "Theoretical study of the erbium-doped fiber laser passively mode-locked by nonlinear polarization rotation”, Phys. Rev. A 67, 013802 (2003).

[30] Leblond, H., Komarov, A., Salhi, M., Haboucha, A. and Sanchez, F., "Cis bound states of three localized pulses of the cubic-quintic CGL equation”, Jour. Opt. A: Pure Appl. Opt. 8, 319-326 (2006).

[31] Komarov, A., Leblond, H. and Sanchez, F., "Multistability and hysteresis phenomena in passively mode-locked fiber lasers", Phys. Rev. A 71, 053809 (2005). 